\title{
Prevalence of Metabolic Syndrome and its Individual Components among Moroccan Adolescents: The Role of Overweight-Obesity and Excess Body Fat
}

\author{
Abdeslam Hamrani ${ }^{*}$, Slimane Mehdad ${ }^{2}$, Khalid El Kari², Asmaa El Hamdouchi ${ }^{2}$, Amina Barkat ${ }^{2,4}$, Hakim Belghiti ${ }^{3}$, Mohammed El Mzibri², \\ Noureddine El Haloui ${ }^{2}$, Najat Mokhtar ${ }^{2}$ and Hassan Aguenaou ${ }^{2}$ \\ 1 Université Ibn Tofaïl, BP 133 Kénitra 14000, Morocco \\ 2Unité Mixte de Recherche en Nutrition \& Alimentation - URAC39 (Université Ibn Tofaïl, BP 133 Kénitra 14000 - Centre National de l'Energie, des Sciences et des \\ Techniques Nucléaires, BP 1382 RP Rabat 10001), Morocco \\ ${ }^{3}$ Unité de nutrition-Service d'hygiène et médecine de collectivité, Hôpital Militaire d'Instruction Mohammed V, Rabat, Morocco \\ ${ }^{4}$ Equipe de Recherche en Santé et Nutrition du Couple Mère-Nouveau-Né, Faculté de Médecine et Pharmacie, Rabat 10001, Morocco
}

\begin{abstract}
Background: The prevalence of obesity and related diseases such as metabolic syndrome (MetS) are increasing in young populations over the world, especially in developing countries. This study aimed to estimate the prevalence of MetS and its individual components, and to assess their association with both overweight/obesity and excess body fat.

Methods: This study included 192 adolescents (77 boys and 115 girls) aged 11 to 17 years. Blood pressure and anthropometric measurements were done using standardized techniques. Fasting blood samples were taken for determination of glucose and lipids levels. Percent body fat was estimated using deuterium oxide. The international diabetes federation criteria were applied for the diagnosis of MetS.

Results: The prevalence of MetS among overweight/obese adolescents was $18.6 \%$ while it was not observed in their normal weight counterparts. The most common abnormality found in the study population was reduced HDLcholesterol (26.6\%) followed by elevated fasting blood glucose (22.4\%) and high waist circumference (19.3\%). Among overweight/obese groups, the rates of individuals with raised TG and reduced HDL-cholesterol were significantly higher in boys than girls ( $35.0 \%$ vs. $15.2 \%, p=0.044$; and $32.5 \%$ vs. $13.0 \%, p=0.038$, respectively). Compared to normal-weight adolescents, overweight/obese subjects had higher odds of having at least two MetS components $(\mathrm{OR}=5.37,95 \% \mathrm{Cl}: 2.72-10.59)$, or at least three MetS components ( $\mathrm{OR}=11.80,95 \% \mathrm{Cl}: 3.40-41.03)$. Excess body fat showed similar degrees of association with clustering MetS components.
\end{abstract}

Conclusions: The prevalence of MetS and its individual components was quite high among the study population, particularly among overweight/obese adolescents. MetS components were strongly associated with both overweight/ obesity and excess body fat. MetS and its components might be best predicted by obesity indexes, determined according to age and body mass index in adolescents, than accurate percent body fat.

Keywords: Blood pressure; Body mass index; Fasting blood glucose; Lipid profile; Waist circumference

Abbreviations: BMI: Body Mass Index; DBP: Diastolic Blood Pressure; FBG: Fasting Blood Glucose; HDL-C: High Density Lipoprotein Cholesterol; LDL-C: Low Density Lipoprotein Cholesterol; MetS: Metabolic Syndrome; OR (95\% CI): Odds Ratio (95\% confidence interval); PBF: Percent Body Fat; SBP: Systolic Blood Pressure; SD: Standard Deviation; TC: Total Cholesterol; TG: Triglycerides; WC: Waist Circumference

\section{Introduction}

The worldwide prevalence of overweight and obesity is increasing rapidly among all age groups [1,2]. According to the International Obesity Task Force (IOTF), at least $10 \%$ of school children aged 5-17 years are overweight or obese, representing a total of 155 million children [3]. It is commonly recognized that obesity is associated with multiple chronic diseases such as diabetes mellitus, hypertension, cardiovascular diseases and dyslipidemia $[4,5]$. The constellation of these metabolic abnormalities and abdominal obesity contribute to the onset of metabolic syndrome (MetS) [6-8], which has become one of the most severe health problems of the $21^{\text {st }}$ century [9].

The prevalence of MetS is growing in young populations, especially in developing countries, and the pediatric MetS predicts adult MetS and type 2 diabetes mellitus [10]. Depending on the criteria used, recent estimates suggest that the population-attributable fraction of MetS is approximately $30 \%-52 \%$ for diabetes, $12 \%-17 \%$ for cardiovascular diseases and 6\%-7\% for all-cause of mortality [11]. Accordingly, the early identification of individuals at risk of developing obesity and MetS is essential for the prevention of morbidity and premature mortality [12].

A set of anthropometric indicators have been shown to be associated with obesity-related diseases, including cardiovascular diseases, diabetes and MetS $[13,14]$. For instance, the current WHO’s Body Mass Index (BMI) ranges were developped empirically to estimate varying levels of morbidity and mortality risks [15]. However, the sensitivity and specificity of BMI ranges in children and adults are often judged using Percent Body Fat (PBF) as the reference $[16,17]$. Also, the PBF, a global and accurate measure of adiposity, may not be an optimal indicator of the health risks among various clinical measures [18]. Thus it is important to further test both BMI and PBF, and determine which one of them is a better marker for obesity-related risks in different ethnic groups.

*Corresponding author: Abdeslam Hamrani, Université Ibn Tofaïl, Faculté des Sciences, Département de Biologie, Laboratoire de Nutrition et Santé, BP 133 Kénitra 14000, Morocco, Tel: 00212668995453; Fax: 00212537711846; E-mail salamhamrani@hotmail.com

Received July 24, 2013; Accepted October 15, 2013; Published October 17 2013

Citation: Hamrani A, Mehdad S, El Kari K, El Hamdouchi A, Barkat A, et al (2013) Prevalence of Metabolic Syndrome and its Individual Components among Moroccan Adolescents: The Role of Overweight-Obesity and Excess Body Fat. J Metabolic Synd 2: 129. doi:10.4172/2167-0943.1000129

Copyright: ( $) 2013$ Hamrani A, et al. This is an open-access article distributed under the terms of the Creative Commons Attribution License, which permits unrestricted use, distribution, and reproduction in any medium, provided the original author and source are credited. 
Citation: Hamrani A, Mehdad S, El Kari K, El Hamdouchi A, Barkat A, et al. (2013) Prevalence of Metabolic Syndrome and its Individual Components among Moroccan Adolescents: The Role of Overweight-Obesity and Excess Body Fat. J Metabolic Synd 2: 129. doi:10.4172/21670943.1000129

Page 2 of 7

To contribute to MetS research that is of fundamental importance for ultimate control and prevention of obesity-related health risks among adolescents, we conducted the current study in Rabat region, Morocco. Our main objectives were to estimate the prevalence of MetS and its individual components; and to assess the association of overweight-obesity and excess body fat with each of MetS components.

\section{Methods}

\section{Study design and participants}

The current study was carried out in the urban area of Rabat region (Morocco). After identifying the participating schools, we sought and obtained the permission to conduct the study from the Ministry of National Education. Students from 10 schools encompassing 680 students were invited to join the study. From each school, 12 classes were randomly chosen representing three grades. The recruitment was started on $28^{\text {th }}$ November, 2008 and finished on $20^{\text {th }}$ March, 2010. In 2010, three schools did not agree to participate in the study. In this year, there has been a national vaccination campaign against the pandemic influenza $\left(\mathrm{A} / \mathrm{H}_{1} \mathrm{~N}_{1}\right)$, which has not been accepted by a large part of the population, and both students and parents thought that the study could have a relationship with this vaccination campaign. A socio-demographic questionnaire was filled out by the parents/legal guardians of the students. On the day of measurements, we accepted only 294 students who signed consents by their parents. After that, we have taken only participants having no missing values in lipid profile measurements, glycemia, blood pressure, WC and age for defining MetS. The final sample consisted of 192 adolescents ( 77 boys and 115 girls) aged 11-17 years, recruited from seven middle schools randomly selected. Given the low proportion of overweight and obese individuals among the targeted population, normal weight and overweight/obese adolescents were separately used as two samples. This separation was based on the BMI values of randomly recruited adolescents according to their age. Informed consent and assent were obtained from all parents and students respectively. A brief relevant clinical examination was done. Participants with physical disability and severe diseases were excluded. The study protocol was approved by the ethic committee from Ibn Tofail University and Ministry of National Education.

\section{General examination}

Anthropometric measurements: Anthropometric measurements were performed by a trained researcher according to Lohman's recommendations [19]. Body weight was taken using a mechanical flat scale $(150 \pm 0.1 \mathrm{~kg})$ with round dial (Seca 750, Germany). Height was measured with a stadiometer (Seca, Germany) to the nearest millimeter $(200 \pm 0.1 \mathrm{~cm})$. BMI in $\mathrm{kg} / \mathrm{m}^{2}$ was calculated as body weight in $\mathrm{kg}$ divided by squared height in $\mathrm{m}^{2}$. Waist circumference was measured to the nearest $0.1 \mathrm{~cm}$ over skin at the midpoint between the lowest rib and the iliac crest and at the end of normal expiration, using a flexible measuring tape. The nutritional status of each subject was categorized using BMI-for-age z-scores based on WHO 2007 growth reference (obese: > +2SD; overweight: > +1SD; and normal weight:-2SD to $+1 \mathrm{SD})[20]$.

Blood pressure: Systolic and diastolic blood pressures (SBP and DBP) were measured by pediatrician using a standard pediatric sphygmomanometer at nearest $2 \mathrm{mmHg}$. The measurement was taken on the right arm, in seated position, after 5-minutes of rest, and in optimal room conditions.

\section{Laboratory analyses}

After an overnight fast, a venous blood sample was collected using the standard venipuncture by a trained nurse. All blood samples were centrifuged at the study sites, plasma was put in separate labeled tubes and transferred immediately in cold boxes filled with ice to the laboratory of the joint research Unit On Nutrition And Food Sciences (URAC 39), Ibn Tofail University and CNESTN, Rabat- Morocco. The samples were stored at $-80^{\circ} \mathrm{C}$ until the day of analysis. All biochemical analyses were carried out by the same team and the same method throughout the study period. Fasting Blood Glucose (FBG) was measured using the hexokinase method [21]. Total Cholesterol (TC) was measured by the enzymatic colorimetric Trinder method [22], High Density Lipoprotein-Cholesterol (HDL-C) was estimated by the PEG 6000 precipitation method [23], and Triglycerides (TG) were estimated by the GPO-PAP-enzymatic colorimetric method [24]. The absorbance was determined by a spectrophotometer (6505 UV/Vis, Jenway). Low Density Lipoprotein Cholesterol (LDL-C) levels were calculated with the Friedwald equation [25] only for subjects having TG levels $<3.75 \mathrm{mmol} / \mathrm{l}$.

PBF was estimated using isotopic technique [26], with the administration of an oral deuterium oxide dose of $99.8 \%{ }^{2} \mathrm{H}_{2} \mathrm{O}(0.5$ g of ${ }^{2} \mathrm{H}_{2} \mathrm{O}$ per kilogram of body weight) to each participant. After an overnight fast, saliva samples (pre-dose, and post-dose after 4 hours) were taken by trained staff. The deuterium enrichment was determined by Fourier Transform Infrared Spectroscopy (FTIR). The body fat was considered excessive when PBF $\geq 25 \%$ in boys and $\geq 30 \%$ in girls [27].

\section{Definition of metabolic syndrome}

MetS was defined according to the International Diabetes Federation (IDF) criteria [28]. For subjects aged $11-16$ years $(10 \leq 16$ yrs), a diagnosis of MetS was made as the presence of abdominal obesity ( $\geq 90^{\text {th }}$ percentile) and the presence of two or more of the following components: raised TG $(\geq 1.7 \mathrm{mmol} / \mathrm{l})$, reduced HDL-C $(<1.03 \mathrm{mmol} / \mathrm{l})$, raised SBP $(\geq 130 \mathrm{mmHg})$ or raised DBP $(\geq 85 \mathrm{mmHg})$, and fasting plasma glucose $(\geq 5.6 \mathrm{mmol} / \mathrm{l})$. The IDF criteria for adults were used to define MetS in those aged 16 years or more ( $\geq 16$ yrs). In this group, the diagnosis of the MetS was made as the presence of abdominal obesity (WC $\geq 94 \mathrm{~cm}$ for boys, and WC $\geq 80 \mathrm{~cm}$ for girls) and the presence of at least two of the following components: raised TG $(\geq 1.7 \mathrm{mmol} / \mathrm{l})$, reduced HDL-C $(<1.03 \mathrm{mmol} / \mathrm{l}$ in boys, and $<1.29 \mathrm{mmol} / \mathrm{l}$ in girls $)$, raised SBP $(\geq 130 \mathrm{mmHg}$ ) or raised DBP $(\geq 85 \mathrm{mmHg})$, fasting plasma glucose $(\geq 5.6 \mathrm{mmol} / \mathrm{l})$

\section{Statistical analysis}

All statistical analyses were carried out using Statistical Package for the Social Sciences (SPSS, version 17.0). The normality of variables' distribution was tested by Kolmogorov-Smirnov test. The variables normally distributed were presented as mean \pm standard deviation, and those non-normally distributed as median (interquartile range). The homogeneity of variances was tested using Levene's test, and the correction of Welch was used in the case of non-homogeneous variances. ANOVA was used to compare variances between independent samples. Mann-Whitney test was performed to compare independent samples for variables non-normally distributed. Chi-square test or Fisher's test were used to test independence between nominal variables. Logistic regression was conducted to test the association of overweight-obesity and excess body fat with MetS and its individual components. P-values less than 0.05 were considered statistically significant.

\section{Results}

This study included a total of 192 subjects ( 77 boys and 115 girls) 
Citation: Hamrani A, Mehdad S, El Kari K, El Hamdouchi A, Barkat A, et al. (2013) Prevalence of Metabolic Syndrome and its Individual Components among Moroccan Adolescents: The Role of Overweight-Obesity and Excess Body Fat. J Metabolic Synd 2: 129. doi:10.4172/21670943.1000129

Page 3 of 7

aged 11 to 17 years with a mean of $14.21 \pm 1.07$ years. Table 1 shows the anthropometric, lipid profile and clinical characteristics of the study population. Among normal-weight group, means of WC and SBP were significantly higher in boys than girls $(\mathrm{p}=0.006$ and $\mathrm{p}=0.023$, respectively), while PBF was significantly higher in girls than boys $(\mathrm{p}=0.006)$. In overweight/obese group, girls showed higher means of BMI and PBF than boys ( $\mathrm{p}=0.026$ and $\mathrm{p}=0.036$, respectively).

The prevalence of MetS among overweight/obese adolescents was $18.6 \%$ while it was not observed in their normal weight counterparts (Table 2). The proportion of subjects with MetS was higher in boys than girls $(20.0 \%$ vs. $17.4 \%)$ with no significant gender difference. Regarding the prevalence of individual components of MetS, the most common abnormality found in the study population was reduced HDL-C (26.6\%) followed by elevated Fasting Blood Glucose (FBG) (22.4\%) and high WC (19.3\%). In normal weight group, the proportion of individuals with elevated FBG was significantly higher among boys than girls ( $27.0 \%$ vs. $17.4 \%, \mathrm{p}=0.011)$. While, among overweight/obese adolescents, the rates of individuals with raised TG and reduced HDL-C were significantly higher in boys than girls $(35.0 \%$ vs. $15.2 \%, \mathrm{p}=0.044$ and $32.5 \%$ vs. $13.0 \%, \mathrm{p}=0.038$, respectively).

Table 3 shows the prevalence of clustering components of MetS and their association with nutritional status and body fat levels. Among the whole study population, the proportions of adolescents with at least one, two and three components of MetS were $64.0 \%, 32.02 \%$ and $13 \%$, respectively. Compared to normal-weight adolescents and to those without excess body fat, both overweight/obese subjects and those with excess body fat had higher odds ratios of having at least one MetS component (OR=2.32 (95\%CI: 1.25-4.30); and OR=1.5 (95\%CI: $0.74-$ 3.03); respectively), at least two MetS components ( $\mathrm{OR}=5.37$ (95\%CI: 2.72-10.59); and $\mathrm{OR}=6.35$ (95\%CI: 2.26-17.82) respectively), or at least three MetS components (OR=11.80(95\%CI: 3.40, 41.03); and $\mathrm{OR}=15.44$ (95\%CI: 1.98, 120.40); respectively).

A logistic regression analysis was performed to evaluate the independent association of overweight-obesity and excess body fat with each component of MetS (Table 4). Among the study population, except for reduced HDL-C, the chance of developing high WC, elevated TG levels, elevated FBG, raised SBP and DBP was higher among overweight/obese adolescents than normal weight individuals, and higher among adolescents with excess body fat than those without excess body fat. Similar results were found in both genders separately except for HDL-C among girls, and FBG among overweight or obese boys. Although most of MetS components showed approximately the same association with both overweightobesity and excess body fat. The degree of this association appeared

\begin{tabular}{|c|c|c|c|c|c|c|c|}
\hline & Total & \multicolumn{3}{|c|}{ Normal weight } & \multicolumn{3}{c|}{ Overweight/obese } \\
\hline & $(\mathrm{n}=192)$ & Boys $(\mathrm{n}=37)$ & Girls $(\mathrm{n}=69)$ & $P$-values & Boys $(\mathrm{n}=40)$ & Girls $(\mathrm{n}=46)$ & $P$-values \\
\hline Age $(\mathrm{years})$ & $14.21 \pm 1.07$ & $14.39 \pm 0.93$ & $14.00 \pm 1.05$ & 0.062 & $13.97 \pm 0.94$ & $14.60 \pm 1.21$ & 0.008 \\
\hline Weight $(\mathrm{kg})$ & $57.45 \pm 14.25$ & $51.33 \pm 9.70$ & $47.56 \pm 7.35$ & 0.026 & $65.86 \pm 13.42$ & $69.90 \pm 12.12$ & 0.145 \\
\hline Height $(\mathrm{m})$ & $1.60 \pm 0.09$ & $1.63 \pm 0.10$ & $1.58 \pm 0.08$ & 0.015 & $1.60 \pm 0.10$ & $1.60 \pm 0.06$ & 0.879 \\
\hline BMI $\left(\mathrm{kg} / \mathrm{m}^{2}\right)$ & $22.33 \pm 4.62$ & $19.27 \pm 2.18$ & $19.01 \pm 2.07$ & 0.556 & $25.37 \pm 3.24$ & $27.13 \pm 3.87$ & 0.026 \\
\hline WC $(\mathrm{cm})$ & $78.66 \pm 12.40$ & $74.05 \pm 8.07$ & $69.48 \pm 8.00$ & 0.006 & $88.08 \pm 10.11$ & $87.93 \pm 10.09$ & 0.944 \\
\hline TC $(\mathrm{mmol} / \mathrm{L})$ & $3.59(2.84-4.64)$ & $3.66(2.91-4.74)$ & $3.49(2.72-4.11)$ & 0.261 & $3.97(2.88-5.42)$ & $3.69(2.98-4.59)$ & 0.334 \\
\hline TG $(\mathrm{mmol} / \mathrm{L})$ & $0.95(0.64-1.41)$ & $0.99(0.63-1.27)$ & $0.77(0.53-1.23)$ & 0.372 & $1.28(0.81-2.08)$ & $0.99(0.64-1.38)$ & 0.043 \\
\hline HDL-C $(\mathrm{mmol} / \mathrm{L})$ & $1.38 \pm 0.54$ & $1.37 \pm 0.47$ & $1.37 \pm 0.60$ & 0.978 & $1.22 \pm 0.51$ & $1.53 \pm 0.51$ & 0.006 \\
\hline LDL-C $(\mathrm{mmol} / \mathrm{L})$ & $1.62(1.13-2.68)$ & $1.53(1.20-2.72)$ & $1.59(1.10-2.33)$ & 0.409 & $2.23(1.24-3.11)$ & $1.61(0.85-2.59)$ & 0.123 \\
\hline FM (kg) & $19.07 \pm 10.00$ & $10.68 \pm 6.02$ & $13.38 \pm 4.95$ & 0.080 & $23.39 \pm 9.68$ & $27.93 \pm 8.77$ & 0.061 \\
\hline PBF $(\%)$ & $31.60 \pm 10.18$ & $19.44 \pm 9.98$ & $27.83 \pm 6.68$ & 0.006 & $35.20 \pm 9.35$ & $39.73 \pm 7.12$ & 0.036 \\
\hline SBP $(\mathrm{mmHg})$ & $114.5(104.3-121.0)$ & $111.0(105.0-120.5)$ & $108.0(100.0-117.5)$ & 0.023 & $120.0(112.8-130.0)$ & $120.0(111.8-130.0)$ & 0.268 \\
\hline DBP $(\mathrm{mmHg})$ & $70.0(60.0-80.0)$ & $70.0(56.00-78.5)$ & $68.0(60.0-70.5)$ & 0.682 & $80.0(70.0-80.0)$ & $70.0(65.75-80.0)$ & 0.069 \\
\hline FBG $(\mathrm{mmol} / \mathrm{L})$ & $2.37(2.18-2.54)$ & $2.41(2.20-2.78)$ & $2.33(2.20-2.44)$ & 0.076 & $2.41(2.24-2.64)$ & $2.34(2.15-2.52)$ & 0.312 \\
\hline
\end{tabular}

Data are presented as mean \pm standard deviation, or median (interquartiles at 25 and 75 )

$P$-values indicate the differences between genders: $P<0.05$, significant difference; $P<0.01$, highly significant difference.

Differences were determined using ANOVA for means, or $U$ Mann-Whitney test for medians.

Table 1: Characteristics of the study population.

\begin{tabular}{|c|c|c|c|c|c|c|c|c|c|}
\hline & \multirow{2}{*}{$\begin{array}{l}\text { Overall } \\
(n=192)\end{array}$} & \multicolumn{4}{|c|}{ Normal weight } & \multicolumn{4}{|c|}{ Overweight/obese } \\
\hline & & $\begin{array}{l}\text { Boys } \\
(n=37)\end{array}$ & $\begin{array}{c}\text { Girls } \\
(n=69)\end{array}$ & $\begin{array}{c}\text { Total } \\
(n=106)\end{array}$ & $P$-values & $\begin{array}{l}\text { Boys } \\
(n=40)\end{array}$ & $\begin{array}{c}\text { Girls } \\
(n=46)\end{array}$ & $\begin{array}{l}\text { Total } \\
(n=86)\end{array}$ & $P$-values \\
\hline High WC (\%) & 19.3 & 2.7 & 0.0 & 0.9 & 0.349 & 47.5 & 37.0 & 41.9 & 0.383 \\
\hline Raised TG (\%) & 16.7 & 10.8 & 10.1 & 10.4 & 0.999 & 35.0 & 15.2 & 24.4 & 0.044 \\
\hline Reduced HDL-C (\%) & 26.6 & 24.3 & 33.3 & 30.2 & 0.381 & 32.5 & 13.0 & 21.1 & 0.038 \\
\hline Raised SBP (\%) & 17.7 & 16.2 & 5.8 & 9.4 & 0.093 & 30.0 & 26.1 & 27.9 & 0.810 \\
\hline Raised DBP (\%) & 8.9 & 0.0 & 2.9 & 1.9 & 0.541 & 17.5 & 17.4 & 17.4 & 0.999 \\
\hline Elevated FBG (\%) & 22.4 & 27.0 & 17.4 & 20.8 & 0.011 & 30.0 & 19.6 & 24.4 & 0.318 \\
\hline MetS (\%) & 8.3 & 0.0 & 0.0 & 0.0 & - & 20.0 & 17.4 & 18.6 & 0.787 \\
\hline
\end{tabular}

Data are presented as proportions

$P$-values indicate the difference between genders

$P<0.05$, significant difference

$P<0.01$, highly significant difference

Differences were determined using Chi-square test or Fisher's test

Table 2: Prevalence of MetS and its individual components among the study population. 
Citation: Hamrani A, Mehdad S, El Kari K, El Hamdouchi A, Barkat A, et al. (2013) Prevalence of Metabolic Syndrome and its Individual Components among Moroccan Adolescents: The Role of Overweight-Obesity and Excess Body Fat. J Metabolic Synd 2: 129. doi:10.4172/21670943.1000129

Page 4 of 7

\begin{tabular}{|c|c|c|c|}
\hline & $\geq 1$ component & $\geq 2$ components & $\geq 3$ components \\
\hline & OR $(95 \% \mathrm{Cl})$ & OR $(95 \% \mathrm{Cl})$ & OR $(95 \% \mathrm{Cl})$ \\
\hline \multicolumn{4}{|l|}{ Overall } \\
\hline \multicolumn{4}{|l|}{ Nutritional status } \\
\hline Normal weight & 1 & 1 & 1 \\
\hline Overweight/obese & $2.32(1.25,4.30)$ & $5.37(2.72,10.59)$ & $11.80(3.40,41.03)$ \\
\hline \multicolumn{4}{|l|}{ Percent body fat } \\
\hline Without excess & 1 & 1 & 1 \\
\hline With excess & $1.50(0.74,3.03)$ & $6.35(2.26,17.82)$ & $15.44(1.98,120.40)$ \\
\hline \multicolumn{4}{|l|}{ Boys } \\
\hline \multicolumn{4}{|l|}{ Nutritional status } \\
\hline Normal weight & 1 & 1 & 1 \\
\hline Overweight/obese & $3.81(1.09,13.30)$ & $6.43(2.28,18.15)$ & - \\
\hline \multicolumn{4}{|l|}{ Percent body fat } \\
\hline Without excess & 1 & 1 & 1 \\
\hline With excess & $4.89(1.00,23.93)$ & $9.75(1.80,52.85)$ & - \\
\hline \multicolumn{4}{|l|}{ Girls } \\
\hline \multicolumn{4}{|l|}{ Nutritional status } \\
\hline Normal weight & 1 & 1 & 1 \\
\hline Overweight/obese & $1.70(0.80 .3 .62)$ & $4.29(1.71,10.73)$ & $6.11(1.58,23.63)$ \\
\hline \multicolumn{4}{|l|}{ Percent body fat } \\
\hline Without excess & 1 & 1 & 1 \\
\hline With excess & $0.97(0.42,2.22)$ & $4.70(1.24,17.91)$ & $6.83(0.80,58.05)$ \\
\hline
\end{tabular}

OR (95\% Cl): Odds Ratio (95\% Confidence Interval) calculated from logistic regression

Table 3: Logistic regression analysis for association of overweight and excess body fat with having at least one component of MetS, having at least two components of MetS, and having at least three components of MetS.

\begin{tabular}{|c|c|c|c|c|c|c|}
\hline & High WC & Raised TG & Reduced HDL-C & Raised SBP & Raised DBP & Elevated FBG \\
\hline & OR $(95 \% \mathrm{Cl})$ & OR $(95 \% \mathrm{Cl})$ & OR $(95 \% \mathrm{Cl})$ & OR $(95 \% \mathrm{Cl})$ & OR $(95 \% \mathrm{Cl})$ & OR $(95 \% \mathrm{Cl})$ \\
\hline \multicolumn{7}{|l|}{ Overall } \\
\hline \multicolumn{7}{|l|}{ Nutritional status } \\
\hline Normal weight & 1 & 1 & 1 & 1 & 1 & 1 \\
\hline Overweight/obese & $75.59(10.08,567.25)$ & $2.79(1.26,6.18)$ & $0.66(0.34,1.27)$ & $3.72(1.66,8.30)$ & $10.99(2.44,49.53)$ & $1.23(0.63,2.44)$ \\
\hline \multicolumn{7}{|l|}{ Percent body fat } \\
\hline Without excess & 1 & 1 & 1 & 1 & 1 & 1 \\
\hline With excess & $10.18(2.27,45.76)$ & $5.31(1.14,24.80)$ & $0.55(0.25,1.20)$ & $3.85(1.34,11.03)$ & $9.76(1.22,78.04)$ & $2.16(0.78,5.97)$ \\
\hline \multicolumn{7}{|l|}{ Girls } \\
\hline \multicolumn{7}{|l|}{ Nutritional status } \\
\hline Normal weight & 1 & 1 & 1 & 1 & 1 & 1 \\
\hline Overweight/obese & - & $1.59(0.52,4.88)$ & $0.30(0.11,0.81)$ & $5.74(1.72,19.14)$ & $7.05(1.42,34.93)$ & $1.62(0.59,4.46)$ \\
\hline \multicolumn{7}{|l|}{ Percent body fat } \\
\hline Without excess & 1 & 1 & 1 & 1 & 1 & 1 \\
\hline With excess & $13.33(1.65,107.70)$ & $4.65(0.52,41.55)$ & $0.25(0.09,0.72)$ & $5.80(1.20,27.93)$ & $3.64(0.39,33.91)$ & $1.58(0.43,5.83)$ \\
\hline \multicolumn{7}{|l|}{ Boys } \\
\hline \multicolumn{7}{|l|}{ Nutritional status } \\
\hline Normal weight & 1 & 1 & 1 & 1 & 1 & 1 \\
\hline Overweight/obese & $32.57(4.06,261.15)$ & $4.44(1.31,15.11)$ & $1.50(0.55,4.08)$ & $2.21(0.73,6.69)$ & - & $0.79(0.30,2.06)$ \\
\hline \multicolumn{7}{|l|}{ Percent body fat } \\
\hline Without excess & 1 & 1 & 1 & 1 & 1 & 1 \\
\hline With excess & $6.59(0.73,59.22)$ & $5.44(0.60,49.56)$ & $1.83(0.45,7.41)$ & $2.25(0.50,10.14)$ & - & $3.06(0.55,16.90)$ \\
\hline
\end{tabular}

OR (95\% Cl): Odds Ratio (95\% Confidence Interval) calculated using logistic regression

Table 4: Logistic regression analysis for association of individual components of MetS with overweight/obesity and excess body fat. 
Citation: Hamrani A, Mehdad S, El Kari K, El Hamdouchi A, Barkat A, et al. (2013) Prevalence of Metabolic Syndrome and its Individual Components among Moroccan Adolescents: The Role of Overweight-Obesity and Excess Body Fat. J Metabolic Synd 2: 129. doi:10.4172/21670943.1000129

Page 5 of 7

to be slightly higher with overweight-obesity than with excess body fat in the total population.

\section{Discussion}

Many studies have produced estimates of MetS prevalence, but there is still insufficient data in several transition countries. To our knowledge, this is the first study addressing the prevalence of MetS and its individual components, and their association with overweight and excess body fat among Moroccan adolescents aged 11 to 17 years. Approximately $8.3 \%$ of all adolescents and $18.6 \%$ of overweight/obese subjects met the IDF criteria for diagnosis of MetS, while none of the normal weight subjects presented MetS. This finding is in agreement with those reported in recent studies indicating higher prevalence of MetS among overweight/obese adolescents compared to their normal-weight counterparts. For instance in some of these studies, the prevalence of MetS among children and adolescents ranged between $11.8 \%$ and $44.0 \%$ in overweight and/or obese groups [6,29-33], while it ranged between $0 \%$ and $4.1 \%$ in the non-overweight groups [34-37]. However, direct comparison is difficult since some of these studies used different definition or cutoff points, some of them worked with large population while others include only overweight and/or obese children and adolescents.

Regarding the gender effect, the prevalence of MetS was slightly higher among boys than girls $(20.0 \%$ vs. $17.4 \%)$ with no significant difference. This result is consistent with many studies conducted elsewhere, such as in USA [2], Latin America [38], Iran [39] and United Arab Emirates [40], which reported higher prevalence of MetS in males than females $(10.8 \%$ vs. $6.1 \%$; $40.0 \%$ vs. $32.2 \%$; $39.7 \%$ vs. $26.3 \% ; 21 \%$ vs. $4 \%$, respectively). This may be due to hormonal differences, such as testosterone and sex hormone binding globulin between genders [41]. Moreover, this can be also explained in our study by the effect of parents' educational level and the household children number.

In the study population, the prevalence of high $\mathrm{WC}$, raised TG, reduced HDL-C, raised SBP, raised DBP and elevated FBG was $19.3 \%$, $16.7 \%, 26.6 \%, 17.7 \%, 8.9 \%$ and $22.4 \%$, respectively. Nearly the same rates for reduced HDL-C (26.1\%) and raised TG (17.2\%) were observed in a sample of 512 Jordanian children and adolescents aged 7-18 years using the IDF criteria [34], whereas in the same study, the rates of elevated FBG (7.2\%) and high blood pressure (6.2\%) were very low. In another study carried out in USA, raised TG and reduced HDL-C were found to be the most prevalent components of MetS among adolescents aged $12-19$ years (25.6\% and $19.3 \%$, respectively), followed by elevated FBG (14.0\%) with prevalence higher among boys than girls (19.8\% vs. $7.9 \%)$ [2]. Our findings differ in some respects from earlier studies, probably due to the difference in proportions of overweight/obese adolescents.

As expected, the proportions of adolescents with individual components of MetS were higher among overweight/obese group than their normal-weight counterparts, except for reduced HDL-C. High WC (41.9\%) was the most common component of MetS, followed by raised SBP (27.9\%), raised TG and elevated FBG (24.4\%). Thus, our data support the notion that overweight and obesity in particular is a great risk for developing the MetS, a finding that is consistent with those reported previously in various ethnic populations $[35,42]$. Our finding also supports that preventive lifestyle interventions should be targeted at lowering both BMI and PBF in Moroccan adolescents.

In addition, among overweight/obese groups, except DBP level which was comparable in both genders, the prevalence of all components of MetS tended to be higher in boys than girls, with significant difference for raised TG and reduced HDL-C. The gender effect on lipid profile has been noted in many studies. For example, the NHANES in USA shown that the prevalence of low HDL-C and raised TG that were higher among males $(24.6 \%, 27.7 \%$ respectively) than females $(13.7 \%$ and $23.3 \%$ respectively) [2]. Also in another study, Daratha and Bindler found higher rates of reduced HDL-C and raised TG among boys than girls ( $25.1 \%$ vs. $20.6 \%$ and $26.6 \%$ vs. $17.5 \%$, respectively) [43]. However, we could not make direct comparison between our data and results of other studies for the raisons mentioned above such us the parents' educational level, and the physical activity levels of adolescents. It is also possible that the gender difference observed in our study could be due to the difference in age between boys and girls (14.0 \pm 0.9 years $v s$. $14.60 \pm 1.21$ years; $\mathrm{p}=0.008$ ) rather than true significance. Haas et al., found in a cohort study over 10 years a decreasing trends for the mean of four components of the MetS, including levels of TG and HDL-C, from childhood to adolescence in both genders [44]. Moreover, the findings of three cohorts, with a total of 678 children aged 8-14 years suggested that evaluation of blood lipid concentrations in children and adolescents should take into account the variation by age and gender [45].

The high rates of adolescents with individual components of MetS among both normal weight and overweight/obese groups may be explained by an unbalanced lifestyle characterized by a regularly consumption of sweet, fatty and starchy foods, and sedentary behavior that lead to increased risks for metabolic disorders [46-50]. Moreover genetics and interaction with environmental factors may increase the risk of developing the MetS [51].

Our results showed that overweight/obesity and excess body fat were associated with increased odds of clustering components of MetS. These findings are consistent with the results of other studies investigating a similar relationship between clustering components of MetS and overweight. It was observed that the prevalence of two MetS components or more was four to six times greater in overweight children and adolescents than in their non-overweight counterparts $[34,46,47]$. Nevertheless, it should be noted that in our study excess body fat was not associated as strongly as overweight/obesity with individual components of MetS. This observation is consistent with that of earlier studies indicating that although PBF is a useful measure of overall adiposity, health risks may be best represented by simply measured BMI $[18,52]$.

Finally, a number of limitations to our analyses need to be considered. First, the relatively small sample size particularly for boys due to the low response rate. Second, the low number of overweight and obese adolescents that limited our ability to consider the two groups separately. These two limitations are mainly due to the low frequency of students who voluntary took and signed consents by their parents, and had no missing values in measurements needed to define MetS among adolescents according to IDF criteria, and no physical disability and severe diseases. The low frequency of the response value by comparison to the reference value in the case of three tests of association using logistic regression has influenced the adjustment validity of three models, this was expressed by missing values of OR and 95\% CI. Third, the prevalence of MetS components could be influenced by other factors such as physical activity and food habits that were not addressed in our study. Despites these limitations, our study provides interesting data that may enable further studies related to MetS and its components for a better understanding of the mechanisms leading to these abnormalities. It could also help the policy-makers and health care practitioners for effective prevention of MetS and its health consequences in adolescents. However, future large-scale studies involving nationally representative samples of adolescents are recommended to make definitive conclusions. 
Citation: Hamrani A, Mehdad S, El Kari K, El Hamdouchi A, Barkat A, et al. (2013) Prevalence of Metabolic Syndrome and its Individual Components among Moroccan Adolescents: The Role of Overweight-Obesity and Excess Body Fat. J Metabolic Synd 2: 129. doi:10.4172/21670943.1000129

In conclusion, the current study provides the first estimates of the prevalence of the MetS and its individual components using the IDF definition among adolescents in Morocco. Our findings revealed high prevalence of these abnormalities particularly among overweight/ obese adolescents. Accordingly, it would be useful to set up urgent strategic intervention to control the growing health and social burdens associated with MetS components, increase the awareness of weightrelated health risks, and promote healthy lifestyle among adolescents. Both overweight/obesity and excess body fat were found to be strongly associated with components of MetS. However, MetS and its components might be best predicted by obesity indexes, determined according to age and BMI in adolescents, than accurate PBF.

\section{Acknowledgements}

We acknowledge and thank all people who dedicated their time and participated in this study. Also, we acknowledge and thank the International Atomic Energy Agency (IAEA) for its technical and financial support.

\section{Authors' Contributions}

Najat Mokhtar, Hassan Aguenaou and Noureddine El Haloui participated in the conception and design of the study, their coordination, revising the manuscript and final approval of the version to be submitted, and provided significant advice all through the study. Najat Mokhtar was the Principal Investigator of the project from which these data were derived. Abdeslam Hamrani and Slimane Mehdad participated in the collection, analysis and interpretation of the data, laboratory analysis, drafting the article. Mohammed El Mzibri, Khalid El Kari and Asmaa El Hamdouchi participated in the collection of data, laboratory analysis, revising the article and final approval of the version to be submitted. Amina Barkat and Hakim Belghiti participated in the collection of data, revising the article and final approva of the version to be submitted.

\section{References}

1. Cali AM, Caprio S (2008) Obesity in children and adolescents. J Clin Endocrinol Metab 93: S31-36.

2. Johnson WD, Kroon JJ, Greenway FL, Bouchard C, Ryan D, et al. (2009) Prevalence of risk factors for metabolic syndrome in adolescents: National Health and Nutrition Examination Survey (NHANES), 2001-2006. Arch Pediatr Adolesc Med 163: 371-377.

3. Lobstein T, Baur L, Uauy R; IASO International Obesity TaskForce (2004) Obesity in children and young people: a crisis in public health. Obes Rev 5 Suppl 1: 4-104.

4. Moran R (1999) Evaluation and treatment of childhood obesity. Am Fam Physician 59: 861-868, 871-3.

5. Cabrinety N, Pisonero MJ, Ajram J, Armenteras A, Cuatrecasas JM (2002) Lipoprotein (a) in obese children with a family history of cardiovascular disease. J Pediatr Endocrinol Metab 15: 77-80.

6. Rizzo AC, Goldberg TB, Silva CC, Kurokawa CS, Nunes HR, et al. (2013) Metabolic syndrome risk factors in overweight, obese, and extremely obese Brazilian adolescents. Nutr J 12: 19.

7. Isomaa B, Almgren P, Tuomi T, Forsén B, Lahti K, et al. (2001) Cardiovascular morbidity and mortality associated with the metabolic syndrome. Diabetes Care 24: 683-689.

8. Lakka HM, Laaksonen DE, Lakka TA, Niskanen LK, Kumpusalo E, et al. (2002) The metabolic syndrome and total and cardiovascular disease mortality in middle-aged men. JAMA 288: 2709-2716.

9. Alvarez Hernández J (2010) Metabolic syndrome. Nutr Hosp Suplementos 3 3-13.

10. Escobedo J, Schargrodsky $H$, Champagne B, Silva H, Boissonnet CP, et al. (2009) Prevalence of the metabolic syndrome in Latin America and its association with sub-clinical carotid atherosclerosis: the CARMELA cross sectional study. Cardiovasc Diabetol 8: 52.

11. Ford ES (2005) Risks for all-cause mortality, cardiovascular disease, and diabetes associated with the metabolic syndrome: a summary of the evidence. Diabetes Care 28: 1769-1778.

12. Ascott-Evans B (2002) The metabolic syndrome, insulin resistance and cardiovascular disease. Cardiovasc J S Afr 13: 187-188.
13. Sayeed MA, Mahtab $\mathrm{H}$, Latif ZA, Khanam PA, Ahsan KA, et al. (2003) Waistto-height ratio is a better obesity index than body mass index and waist-to-hip ratio for predicting diabetes, hypertension and lipidemia. Bangladesh Med Res Counc Bull 29: 1-10.

14. Czernichow S, Kengne AP, Huxley RR, Batty GD, de Galan B, et al. (2011) Comparison of waist-to-hip ratio and other obesity indices as predictors of cardiovascular disease risk in people with type-2 diabetes: a prospective cohort study from ADVANCE. Eur J Cardiovasc Prev Rehabil 18: 312-319.

15. [No authors listed] (2000) Obesity: preventing and managing the global epidemic. Report of a WHO consultation. World Health Organ Tech Rep Ser 894: i-xii, 1-253.

16. Goh VH, Tain CF, Tong TY, Mok HP, Wong MT (2004) Are BMI and other anthropometric measures appropriate as indices for obesity? A study in an Asian population. J Lipid Res 45: 1892-1898.

17. Neovius MG, Linné YM, Barkeling BS, Rossner SO (2004) Sensitivity and specificity of classification systems for fatness in adolescents. Am J Clin Nutr 80: 597-603.

18. Shen W, Punyanitya M, Chen J, Gallagher D, Albu J, et al. (2006) Waist circumference correlates with metabolic syndrome indicators better than percentage fat. Obesity (Silver Spring) 14: 727-736.

19. Lohman TG, Roche AF, Martorell R (1988) Anthropometric standardization reference manual. Champaign, IL: Human Kinetics.

20. World Health Organization (2007) Growth reference 5-19 years

21. Schmidt FH (1973) [Blood glucose levels in capilary blood of adults assessed by the hexokinase method (author's transl)]. Klin Wochenschr 51: 520-522.

22. Alberti G, Zimmet P, Shaw J, Bloomgarden Z, Kaufman F, et al. (2004) Type 2 diabetes in the young: the evolving epidemic: the international diabetes federation consensus workshop. Diabetes Care 27: 1798-1811.

23. World Health Organization (2004) Global strategy on diet, physical activity and health.

24. Tietz NW (1990) Clinical Guide to Laboratory Tests. (3rdedn.), W.B. Saunders Company, Philadelphia, USA.

25. Friedewald WT, Levy RI, Fredrickson DS (1972) Estimation of the concentration of low-density lipoprotein cholesterol in plasma, without use of the preparative ultracentrifuge. Clin Chem 18: 499-502.

26. International Atomic Energy Agency (2009) Assessment of Body Composition and Total Energy Expenditure in Humans by Stable Isotope Techniques. IAEA Human Health Series No 3.

27. Williams DP, Going SB, Lohman TG, Harsha DW, Srinivasan SR, et al. (1992) Body fatness and risk for elevated blood pressure, total cholesterol, and serum lipoprotein ratios in children and adolescents. Am J Public Health 82: 358-363.

28. Alberti SG, Zimmet P, Kaufman F, Tajima N, Silink M, et al. (2007) The IDF consensus definition of the metabolic syndrome in children and adolescents. International Diabetes Federation.

29. Nguyen TH, Tang HK, Kelly P, van der Ploeg HP, Dibley MJ (2010) Association between physical activity and metabolic syndrome: a cross sectional survey in adolescents in Ho Chi Minh City, Vietnam. BMC Public Health 10: 141.

30. Hassan NE, El-Masry SA, Fouad WA, Sherifc L, Elwakkadd A, et al. (2011) Prevalence of metabolic syndrome among obese school students. e SPEN Eu e J Clin Nutr Metab 6:e248-e252.

31. El-Hazmi MA, Warsy AS (2002) A comparative study of prevalence of overweight and obesity in children in different provinces of Saudi Arabia. J Trop Pediatr 48: 172-177.

32. Invitti C, Maffeis C, Gilardini L, Pontiggia B, Mazzilli G, et al. (2006) Metabolic syndrome in obese Caucasian children: prevalence using WHO-derived criteria and association with nontraditional cardiovascular risk factors. Int $\mathrm{J}$ Obes (Lond) 30: 627-633.

33. Atabek ME, Pirgon O, Kurtoglu S (2006) Prevalence of metabolic syndrome in obese Turkish children and adolescents. Diabetes Res Clin Pract 72: 315-321.

34. Khader Y, Batieha A, Jaddou H, El-Khateeb M, Ajlouni K (2010) Metabolic Syndrome and its Individual Components among Jordanian Children and Adolescents. Int J Pediatr Endocrinol 2010: 316170.

35. Wee BS, Poh BK, Bulgiba A, Ismail MN, Ruzita AT, et al. (2011) Risk of metabolic syndrome among children living in metropolitan Kuala Lumpur: a case control study. BMC Public Health 11: 333 
Citation: Hamrani A, Mehdad S, El Kari K, El Hamdouchi A, Barkat A, et al. (2013) Prevalence of Metabolic Syndrome and its Individual Components among Moroccan Adolescents: The Role of Overweight-Obesity and Excess Body Fat. J Metabolic Synd 2: 129. doi:10.4172/21670943.1000129

Page 7 of 7

36. Camhi SM, Kuo J, Young DR (2008) Identifying adolescent metabolic syndrome using body mass index and waist circumference. Prev Chronic Dis 5: A115

37. Esmaillzadeh A, Mirmiran P, Azadbakht L, Etemadi A, Azizi F (2006) High prevalence of the metabolic syndrome in Iranian adolescents. Obesity (Silver Spring) 14: 377-382.

38. Caceres M, Teran CG, Rodriguez S, Medina M (2008) Prevalence of insulin resistance and its association with metabolic syndrome criteria among Bolivian children and adolescents with obesity. BMC Pediatr 8: 31.

39. Ghergherehchi R (2008) Prevalence of metabolic syndrome in obese children and adolescents between 4 and 18 years of age. Arch Dis Child 93: 567

40. Mehairi AE, Khouri AA, Naqbi MM, Muhairi SJ, Maskari FA, et al. (2013) Metabolic syndrome among Emirati adolescents: a school-based study. PLoS One 8: e56159.

41. Agirbasli M, Cakir S, Ozme S, Ciliv G (2006) Metabolic syndrome in Turkish children and adolescents. Metabolism 55: 1002-1006

42. Elizondo-Montemayor L, Serrano-González M, Ugalde-Casas PA, CuelloGarcía C, Borbolla-Escoboza JR (2010) Metabolic syndrome risk factors among a sample of overweight and obese Mexican children. J Clin Hypertens (Greenwich) 12: 380-387.

43. Daratha KB, Bindler RC (2009) Effects of individual components, time, and sex on prevalence of metabolic syndrome in adolescents. Arch Pediatr Adolesc Med 163: 365-370
44. Haas GM, Bertsch T, Schwandt P (2012) Trends of Components of the Metabolic Syndrome in German First Graders Throughout 10 Years: The PEP Family Heart Study. Cholesterol 2012: 231962

45. Dai S, Fulton JE, Harrist RB, Grunbaum JA, Steffen LM, et al. (2009) Blood lipids in children: age-related patterns and association with body-fat indices: Project HeartBeat! Am J Prev Med 37: S56-64.

46. Chu NF, Rimm EB, Wang DJ, Liou HS, Shieh SM (1998) Clustering of cardiovascular disease risk factors among obese schoolchildren: the Taipel Children Heart Study. Am J Clin Nutr 67: 1141-1146.

47. Csábi G, Török K, Jeges S, Molnár D (2000) Presence of metabolic cardiovascular syndrome in obese children. Eur J Pediatr 159: 91-94.

48. Mokhtar N, Elati J, Chabir R, Bour A, Elkari K, et al. (2001) Diet culture and obesity in northern Africa. J Nutr 131: 887S-892S.

49. Rguibi M, Belahsen R (2007) Prevalence of obesity in Morocco. Obes Rev 8: 11-13.

50. Pan Y, Pratt CA (2008) Metabolic Syndrome and its association with diet and physical activity in US adolescents. J Am Diet Assoc 108: 276-286.

51. Wulan SN, Westerterp KR, Plasqui G (2010) Ethnic differences in body composition and the associated metabolic profile: a comparative study between Asians and Caucasians. Maturitas 65: 315-319.

52. Mooney SJ, Baecker A, Rundle AG (2013) Comparison of anthropometric and body composition measures as predictors of components of the metabolic syndrome in a clinical setting. Obes Res Clin Pract 7: e55-e66. 